Bicocca/FT October 2000

\title{
A remark on the numerical validation of triviality for scalar field theories using high-temperature expansions
}

\author{
P. Butera and M. Comi \\ Istituto Nazionale di Fisica Nucleare \\ Dipartimento di Fisica, Università di Milano-Bicocca \\ 3 Piazza della Scienza, 20126 Milano, Italy \\ E-mail:butera@mib.infn.it ; comi@mib.infn.it
}

\begin{abstract}
We suggest a simple modification of the usual procedures of analysis for the high-temperature (strong-coupling or hopping-parameter) expansions of the renormalized four-point coupling constant in the $\vec{\phi}_{4}^{4}$ lattice scalar field theory. As a result we can more convincingly validate numerically the triviality of the continuum limit taken from the high temperature phase.
\end{abstract}

There has been a steady accumulation of suggestive numerical and analytical evidence, but not yet a complete rigorous proof that the continuum limit of the $N$-component $\vec{\phi}_{4}^{4}$ theory, (defined on a lattice and with ferromagnetic nearest-neighbor couplings), describes a free [1 1 (or "trivial") field theory. The basic clues of this paradoxical no-interaction property were clearly indicated almost half a century ago [5], but more stringent studies of this conjecture had to wait for the developments of the Renormalization Group(RG) theory [6]. The modern rigorous analyses of Refs. [1, 2, [10] have finally proved that the $\vec{\phi}_{d}^{4}$ theory is non-trivial [11] in $d \leq 3$ and trivial in $d \geq 5$ dimensions, at least for not too large values of $N$. Triviality is expected to occur (and is supported by numerical calculations) also in $d=4$ dimensions. Since, however, the rigorous results in this direction are still partial, various routes [12,13] to recover an interesting continuum theory have also been explored.

The lattice Euclidean $\vec{\phi}_{4}^{4}$ theory with $O(N)$ symmetry is defined by the action [14]

$$
S=\sum_{x}\left\{-\beta \sum_{\mu} \vec{\phi}_{x} \cdot \vec{\phi}_{x+\mu}+\vec{\phi}_{x}^{2}+\lambda\left(\vec{\phi}_{x}^{2}-1\right)^{2}\right\}
$$

where $\vec{\phi}_{x}$ is a real $N$-component field at the lattice site $x, \mu$ is the unit vector in the $\mu$ direction and $\lambda \geq 0$.

For $\beta \uparrow \beta_{c}(N, \lambda)$, at fixed positive $\lambda$ the model has a critical point where a second order transition occurs from a high-temperature(HT) paramagnetic phase to a low-temperature ferromagnetic phase. In the $\lambda \rightarrow \infty$ limit, the model becomes to the lattice non-linear $O(N)$-symmetric $\sigma$-model or, equivalently, the $N$-vector spin model.

The construction of a continuum limit of the lattice theory is reduced to the determination of its critical properties. Here we shall consider only the continuum limit taken from the HT phase. In the context of the RG theory a detailed description is obtained for the 
asymptotic cutoff dependence of the correlation functions in terms of the weak-coupling expansion of the theory's beta function.

If we set $\tau(N, \lambda)=1-\beta / \beta_{c}(N, \lambda)$, the perturbative RG theory yields [15] the following critical behavior as $\tau \downarrow 0$, for the correlation length

$$
\xi^{2}(\beta, N, \lambda)=A_{\xi}^{2}(N) \frac{|\ln (\tau(N, \lambda))|^{G(N)}}{\tau(N, \lambda)}[1+O(\ln (|\ln (\tau)|) / \ln (\tau))]
$$

where $G(N)=\frac{N+2}{N+8}$.

The asymptotic behavior of the susceptibility is completely similar

$$
\chi(\beta, N, \lambda)=A_{\chi}(N) \frac{|\ln (\tau(N, \lambda))|^{G(N)}}{\tau(N, \lambda)}[1+O(\ln (|\ln (\tau)|) / \ln (\tau))] .
$$

The fourth derivative of the free energy at zero field $\chi_{4}(\beta, N, \lambda)$ has the behavior

$$
\chi_{4}(\beta, N, \lambda)=A_{4}(N) \frac{|\ln (\tau(N, \lambda))|^{4 G(N)-1}}{\tau(N, \lambda)^{4}}[1+O(\ln (|\ln (\tau)|) / \ln (\tau))] .
$$

In terms of $\chi, \xi^{2}$ and $\chi_{4}$, the dimensionless renormalized 4-point coupling constant $g_{r}(N, \lambda)$ is defined by the critical value of the ratio

$$
g_{r}(\beta, N, \lambda)=-\frac{\chi_{4}(\beta, N, \lambda)}{\xi^{4}(\beta, N, \lambda) \chi^{2}(\beta, N, \lambda)}
$$

as $\tau \downarrow 0$.

It can be shown that $g_{r}(\beta, N, \lambda)$ is non negative [16] for all $\beta$. If $g_{r}(\beta, N, \lambda)$ vanishes as $\tau \downarrow 0$, the continuum limit of the lattice model taken from the high-temperature phase describes a (generalized)-free-field theory [17], namely a theory where the connected parts of the four-point and higher-point functions vanish.

As $\tau \downarrow 0$, the perturbative RG yields the leading asymptotic behavior, with a well specified universal amplitude [14]

$$
g_{r}(\beta, N, \lambda) \approx \frac{c(N)}{|\ln (\tau)|}[1+O(\ln (|\ln (\tau)|) / \ln (\tau))]
$$

where $c(N)=2 / b_{1}(N)$ and $b_{1}(N)=\frac{N+8}{48 \pi^{2}}$ is the first non-vanishing coefficient of the betafunction.

Therefore the perturbative RG theory implies that $g_{r}(\beta, N, \lambda) \rightarrow 0$ as $\tau \downarrow 0$, namely that the continuum $\vec{\phi}_{4}^{4}$ model is trivial.

Since the validity of the results in eqs.(2), (3), (㺼), (6) is based upon the (unwarranted) perturbative determination of the beta function, it is interesting, at least, to try to confirm them within different approximation schemes. To this purpose, various HT or strongcoupling expansion analyses [6,14,18 22, have been performed. Many extensive MonteCarlo (MC) lattice simulations 23 31 have also been carried out and progressively refined in the years, following the rapid evolution of computers and the improvement of algorithms and data analysis. Until now, both the stochastic simulation and the HT series studies have been generally carried out in a completely parallel way. For instance, in the case of the 4d 
self-avoiding walk model (namely, the $\vec{\phi}_{4}^{4}$ model for $N=0$ and $\lambda \rightarrow \infty$ ) a HT expansion of $\chi$ up to order $\beta^{13}$ on the hypercubic lattice has been analyzed [18] in order to detect the logarithmic factor predicted by the RG theory eq.(3) and to estimate its exponent. For the same purpose, $\chi$ and $\xi^{2}$ have been measured 23, 30 in high precision MC simulations. Analogous studies [19,21] have been devoted to the $4 \mathrm{~d}$ Ising model (namely, the $N=1$ case for $\lambda \rightarrow \infty)$ using series $\mathrm{O}\left(\beta^{17}\right)$ for $\chi$ and $\chi_{4}$ on the hypercubic lattice. The MC simulations of Refs. [24, 25, 28] have tried to show directly the consistency of the estimates of $g_{r}(\beta, N, \lambda)$ with the elusive asymptotic behavior eq.(6). A somewhat different approach, based on the scaling properties of the partition function zeroes in the complex $\beta$ plane, has been adopted in the simulations of Ref. [31]. Moreover various analytical or semianalytical approaches 32,33 have also been pursued.

All of these non-perturbative calculations have given results consistent, or at least not in contrast, with the predicted critical behaviors of $\chi, \xi$, and $g_{r}$. However, the cited computations are somewhat limited in their extent, since only the $N=0$ and $N=1$ cases for $\lambda \rightarrow \infty$, and, the $N=1$ case for finite $\lambda$ to order $\beta^{10}$ have been considered [20] until now. Moreover, it is a common experience how difficult it is to uncover numerically a logarithmic behavior or, more generally, a logarithmic correction to a power behavior. Indeed, as the computations proceed deeply into the asymptotic regime their reliability decreases and the uncertainties of their results often reach almost the same order of magnitude as the effects that have to be revealed. In the case of the HT expansions, we have also observed that, the methods of Refs. [18, 21] which were very effective in the $N=0$ and $N=1$ cases, are not as successful when $N>1$.

In this note, we do not present new data, but reconsider the HT expansions calculated through order $\beta^{14}$, more than a decade ago, by Lüscher and Weisz [14] for $\chi, \xi^{2}$ and $\chi_{4}$ on the hypercubic lattice. They had produced and analysed these series to obtain a bound on the Higgs particle mass as a consequence of the triviality of the scalar sector in the standard model. They used from the outset also the assumption of validity of the perturbative RG and therefore avoided to place too much confidence in the HT series within the critical regime. We make no such assumption, but rather we suggest a slightly different and hopefully more convincing way of analyzing the series, which takes advantage of the specific smoothness features of the HT expansion approach and, in the end, also turns out to be completely consistent with the RG results. We study how accurately an obvious consequence of eq.(6), rather than the equation itself, is confirmed by computations.

We observe that eq.(6) implies that

$$
F(\beta, N, \lambda)=\tau(N, \lambda) \frac{d}{d \beta}\left(\frac{1}{g_{r}(\beta, N, \lambda)}\right)=\frac{b_{1}(N)}{2}+O\left(\ln (|\ln (\tau)|) / \ln ^{3}(\tau)\right)
$$

as $\tau \downarrow 0$.

In order to confirm triviality, one has then to show at least, that $F(\beta, N, \lambda)$ has a finite limit $\tilde{F}(N, \lambda)$, as $\tau \downarrow 0$. The analysis will be even more compelling if

i) $\tilde{F}(N, \lambda) \approx \tilde{F}(N)$, namely if the quantity $\tilde{F}(N, \lambda)$ will appear not to depend on $\lambda$, as required by universality,

and if, moreover,

ii) $\frac{2 \tilde{F}(N)}{b_{1}(N)} \approx 1$, namely if, unlike in previous approaches, it is possible to show complete quantitative consistency between the strong-coupling estimate of $g_{r}$, including the universal 
amplitude $2 / b_{1}(N)$, and the weak-coupling RG prediction eqs.(6),(7).

Since the HT series can be simply written 34 as explicit functions of $N$, we can easily repeat the analysis on a wide range of values of $N$ and thus further corroborate this result.

Let us stress that the whole analysis cannot be easily performed in the context of a MC simulation, whereas it is completely obvious in a HT series approach.

The main results of our procedure can be summarized into a couple of figures. In Fig.1, for $N=4$, we have plotted the quantity $y=\frac{2 \tilde{F}(\beta, N, \lambda)}{b_{1}(N)}$ versus the scaled variable $x=\beta / \beta_{c}(N, \lambda)$, in order to be able to compare the curves obtained for various fixed values of $\lambda$. The values of $\beta_{c}(N, \lambda)$ used here have been estimated by an analysis of the susceptibility expansions. We have calculated $F(\beta, N, \lambda)$ by simply forming Padé Approximants (PA) of its HT expansion. For each value of $\lambda$, we have plotted only the highest non-defective PA, namely the [6/6] or the $[6 / 7]$ approximants, as appropriate. The other approximants of sufficiently high order have the same behavior and are not reported in the figure. As $x \rightarrow 1$, the various curves so obtained appear to tend to unity, independently of $\lambda$, within a good approximation, thus confirming i). We expect that the residual small spread of the limiting values would be significantly reduced if we could further reduce the uncertainties in the determination of $\beta_{c}(N, \lambda)$ and if we could devise approximants more accurately allowing for the slowly vanishing corrections to scaling indicated in eq.(17). Of course these improvements are strictly related.

We have performed the above calculation for various values of $N$ and, in Fig.2, we have plotted versus $N$ the ratio $R(N)=\frac{2 \tilde{F}(N)}{b_{1}(N)}$. For each value of $N$ the reported error reflects the spread of this quantity. Within a fair approximation, $R(N)$ appears to be unity over a wide range of values of $N$, thus confirming ii).

Therefore both graphs indicate a good quantitative agreement with the asymptotic formula eq.(局), obtained within the perturbative $R G$ approach. These very general results are unlikely to be accidental and completely confirm the conventional expectations concerning triviality.

The HT series we have used in this first test are definitely too short to make a more refined analysis possible. The favourable results, however, suggest that this study should be resumed as soon as our systematic work [35] of HT series extension by the linked-cluster method will make new longer expansions available. We do not expect results qualitatively different from this preliminary study, but significant quantitative improvements.

In conclusion, we have shown that a small modification of current procedures of numerical analysis is sufficient to shift the emphasis from a difficult qualitative question, namely how accurately an elusive logarithmic behavior is reproduced by an approximation scheme of inevitably limited precision, to a more quantitative issue. Our technique of analysis for HT series is not more involved than the usual ones, while, for all values of $N$, it seems to produce a more convincing numerical validation of the perturbative-RG triviality predictions within the strong-coupling approach. 


\section{REFERENCES}

[1] A. Sokal, Ann. Inst. H. Poincaré A 37, 317 (1982).

[2] A. Fernandez, J. Fröhlich and A. Sokal, Random walks, critical phenomena and triviality in quantum field theory, Springer Verlag, Berlin 1992.

[3] K. Symanzik, Journ. de Phys.(France) 43, Suppl. C3, 254 (1982).

[4] D. Callaway, Phys. Rept. 167, 241 (1988)

[5] L. D. Landau and I.Pomeranchuk, Dokl. Akad. Nauk. SSSR 102, 489 (1955); I.Pomeranchuk,V. V. Sudakov and K. A. Ter Martirosian, Phys. Rev. 103, 784 (1956).

[6] K. Wilson and J. B. Kogut, Phys. Rep. C12, 75 (1974).

[7] M. Aizenmann, Phys. Rev. Lett. 47, 1 (1981).

[8] J. Fröhlich, Nucl. Phys. B 200, 281 (1982).

[9] T. Hara, J. Stat. Phys. 47, 57 (1987); T. Hara and H. Tasaki, ibid. 47, 99 (1987).

[10] S.B. Shlosman, Sov. Phys. Dokl. 33, 905 (1988).

[11] J.P.Eckmann and R. Epstein, Comm. Math. Phys. 64,95 (1979).

[12] G. Gallavotti and V. Rivasseau, Ann. Inst. H. Poincaré 40, 185 (1984).

[13] P. Cea. M. Consoli, L. Cosmai and P.M. Stevenson, Mod.Phys.Lett. A 14, 1673 (1999) and References therein.

[14] M. Lüscher and P.Weisz, Nucl. Phys. B 300, 325 (1988); Nucl. Phys. B 290, 25 (1987).

[15] E. Brezin, J.C. Le Guillou and J. Zinn-Justin, in Phase Transitions and Critical Phenomena, vol. VI, ed. C. Domb and M.S. Green, Academic, New York 1976; J. ZinnJustin, Quantum field theory and critical phenomena (Clarendon, Oxford, 1989, third edition 1996).

[16] J. L. Lebowitz, Comm. Math. Phys. 35, 87 (1974).

[17] C. M. Newman, Comm.Math Phys. 41, 1 (1975).

[18] A. J. Guttmann, J. Phys. A 11, 1103 (1978).

[19] D.S. Gaunt, M. F. Sykes and S. McKenzie, J. Phys. A 12, 871 (1979).

[20] G. A. Baker and J.M. Kincaid, Phys. Rev. Lett. 42, 1431 (1979); ibid. (E) 44, 434 (1980); J. Stat. Phys. 24, 469 (1981).

[21] A. Vladikas and C.C. Wong, Phys. Lett. B 189, 154 (1987).

[22] C.M. Bender, F. Cooper, G.S. Guralnik, R. Roskies and D. H. Sharp, Phys. Rev. D 23, 2976(1981); ibid. D 23, 2999(1981); G.A. Baker L.P.Benofy, F. Cooper and D. Preston, Nucl. Phys. B 210273 (1982). C.M. Bender and H. F. Jones, Phys. Rev. D 38, 2526 (1988).

[23] C. A. de Carvalho, J. Fröhlich and S. Caracciolo, Nucl. Phys. B 215 [FS7], 209, (1983).

[24] B. Freedman, P. Smolenski and D. Weingarten, Phys. Lett. B 113, 481 (1982).

[25] I.A. Fox and I. G. Halliday, Phys. Lett. B 159, 148 (1985).

[26] I.T. Drummond, S. Duane and R. R. Horgan, Nucl. Phys. B 280, 25 (1987).

[27] W. Bernreuther, M. Göckeler and M. Kremer, Nucl. Phys. B 295 [FS21], 211(1988).

[28] Ch. Frick, K. Jansen, J. Jersak, I. Montvay, G. Münster and P. Seuferling, Nucl. Phys. $B 331,515(1990)$.

[29] J.K.Kim and A. Patrascioiu, Phys. Rev. D 47, 2588 (1992).

[30] P. Grassberger, R. Hegger and L. Schäfer, J. Phys. A 27, 7265 (1994).

[31] R. Kenna and C. B. Lang, Phys. Rev. E 49, 5012 (1994).

[32] W. Bardeen and M. Moshe, Phys. Rev. D 28, 1372 (1983).

[33] D. Gromes, Z. für Phys. C 71, 347 (1996). 
[34] P.Butera, M. Comi and G. Marchesini, Nucl. Phys. B 300, 1 (1988) and Phys. Rev. B 41, 11494 (1990).

[35] P. Butera, R. Cabassi, M. Comi, G. Marchesini, Comp. Phys. Comm. 44, 143 (1987); P. Butera and M.Comi, Phys. Rev. B 46, 11141 (1992); ibid. B 47, 11969 (1993); ibid. $B$ 50, 3052 (1994); ibid. B 52, 6185 (1995); ibid. B 54, 15828 (1996); ibid. E 55, 6391 (1997); ibid. B 56, 8212 (1997); ibid. B 58, 11552 (1998); ibid. B 60, 6749 (1999); ibid. B 62, 14837 (2000) Nucl. Phys.B ( Proc. Suppl.) 63 A-C, 643 (1998); hep-lat/0006009. 


\section{FIGURES}

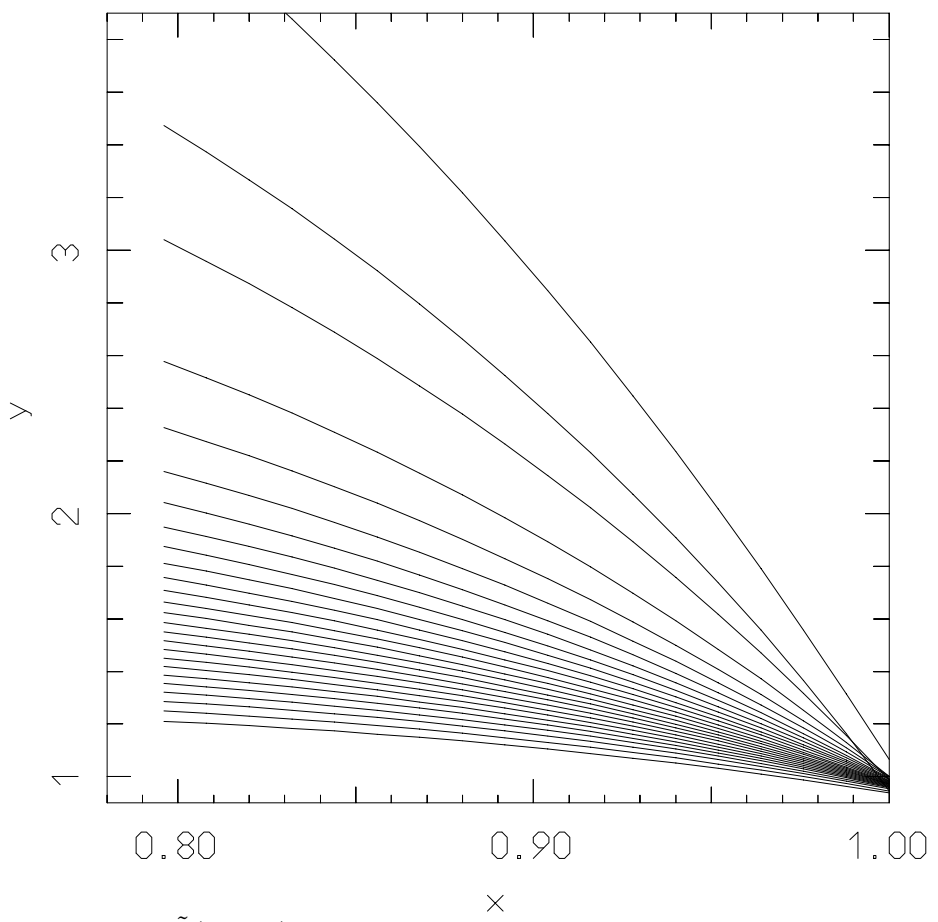

FIG. 1 . The quantity $y=\frac{2 \tilde{F}(\beta, N, \lambda)}{b_{1}(N)}$ versus the scaled variable $x=\beta / \beta_{c}(N, \lambda)$. We have taken $N=4$. Going from the top to the bottom of the figure, the various curves correspond to increasing values of $\lambda$ between 0 and $\infty$. 


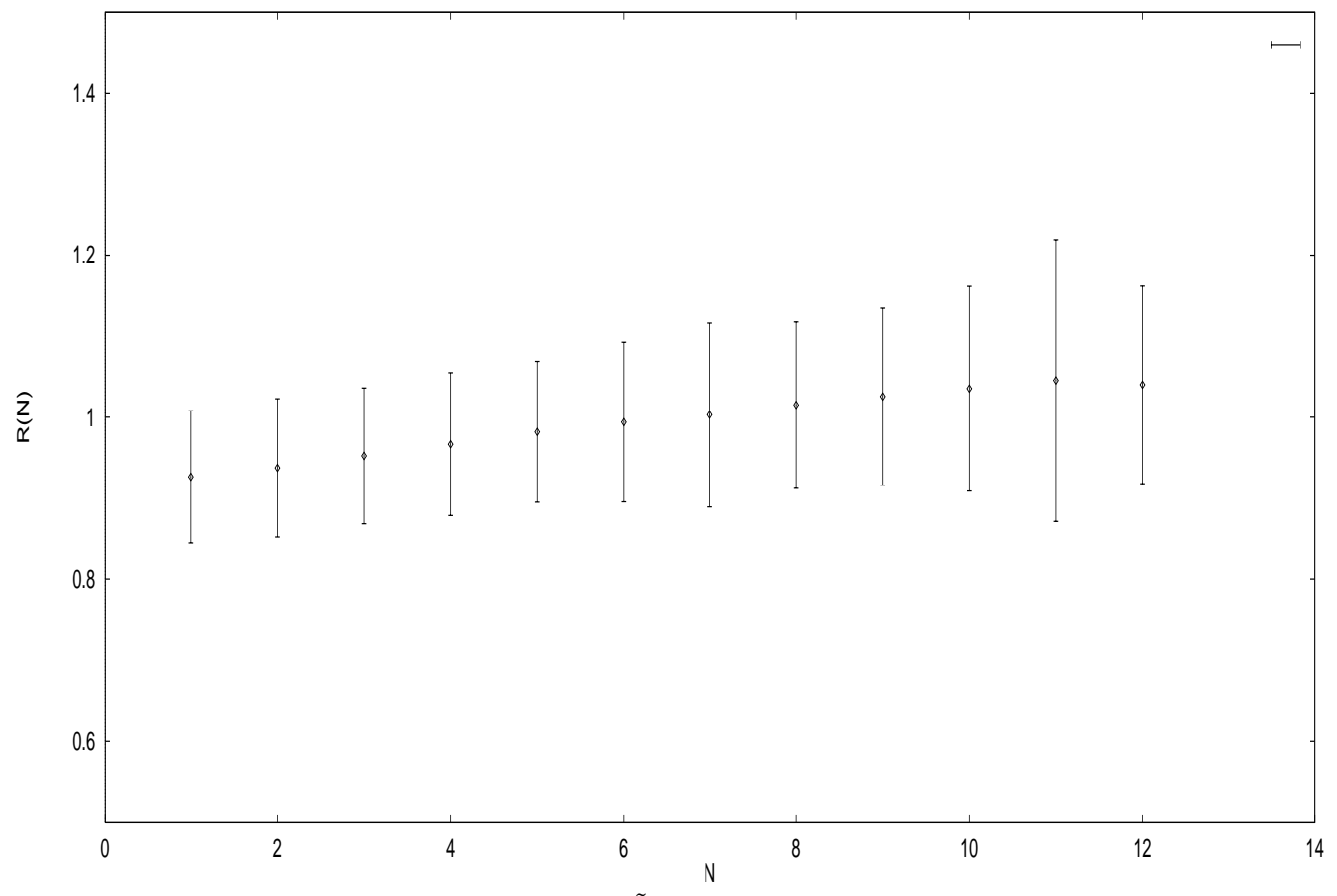

FIG. 2. The ratio $R(N)=\frac{2 \tilde{F}(N)}{b_{1}(N)}$ versus $N$. 